\title{
Development of a Computer Assisted Gantry System for Gaining Rapid and Accurate Calyceal Access during Percutaneous Nephrolithotomy
}

\author{
A. D. Zarrabi, J. P. Conradie, C. F. Heyns, C. Scheffer, K. Schreve \\ Department of Urology (ADZ, CFH), University of Stellenbosch and Tygerberg Hospital, Tyger- \\ berg, South Africa and Department of Mechanical and Mechatronic Engineering (JPC, CS, KS), \\ University of Stellenbosch, Stellenbosch, South Africa
}

\begin{abstract}
Purpose: To design a simple, cost-effective system for gaining rapid and accurate calyceal access during percutaneous nephrolithotomy (PCNL).

Materials and Methods: The design consists of a low-cost, light-weight, portable mechanical gantry with a needle guiding device. Using C-arm fluoroscopy, two images of the contrast-filled renal collecting system are obtained: at 0-degrees (perpendicular to the kidney) and 20-degrees. These images are relayed to a laptop computer containing the software and graphic user interface for selecting the targeted calyx. The software provides numerical settings for the 3 axes of the gantry, which are used to position the needle guiding device. The needle is advanced through the guide to the depth calculated by the software, thus puncturing the targeted calyx. Testing of the system was performed on 2 target types: 1) radiolucent plastic tubes the approximate size of a renal calyx (5 or $10 \mathrm{~mm}$ in diameter, $30 \mathrm{~mm}$ in length); and 2) foam-occluded, contrast-filled porcine kidneys.

Results: Tests using target type 1 with $10 \mathrm{~mm}$ diameter $(\mathrm{n}=14)$ and $5 \mathrm{~mm}$ diameter $(\mathrm{n}=7)$ tubes resulted in a $100 \%$ targeting success rate, with a mean procedure duration of 10 minutes. Tests using target type $2(n=2)$ were both successful, with accurate puncturing of the selected renal calyx, and a mean procedure duration of 15 minutes.

Conclusions: The mechanical gantry system described in this paper is low-cost, portable, light-weight, and simple to set up and operate. C-arm fluoroscopy is limited to two images, thus reducing radiation exposure significantly. Testing of the system showed an extremely high degree of accuracy in gaining precise access to a targeted renal calyx.
\end{abstract}

Key words: nephrolithotomy; percutaneous; access; computers; endourology; urolithiasis

Int Braz J Urol. 2010; 36: 738-48

\section{INTRODUCTION}

Since the first description of percutaneous nephrolithotomy (PCNL) by Fernström and Johansson more than 30 years ago, major technological advances have improved the efficacy and safety of this procedure, confirming its superiority compared to open surgery for renal calculi (1).
Obtaining precise access to a predetermined renal calyx is the most critical part of PCNL (2). Currently available techniques for obtaining percutaneous (PC) access to the renal collecting system include the following:

Two-stage procedure: Pre-operative access is first obtained by an interventional radiologist using ultrasound guidance, after which the urologist dilates 
the tract and performs the PCNL. A recent report indicates that the minority of urologists $(11 \%)$ gain their own access for PCNL (3).

Retrograde percutaneous access: This involves retrograde placement of a ureteric catheter, followed by passage of a sharp wire through the catheter and via the selected calyx to the skin (4). Despite the feasibility of this method, it offers no advantage over antegrade percutaneous access and is not commonly utilized.

Fluoroscopic X-ray guided techniques: The two techniques best described are "eye of the needle" and "triangulation". Both consist of several steps where $\mathrm{C}$-arm fluoroscopy is rotated into different positions relative to the needle and the target (contrast-filled calyx) (2). The needle is advanced until the calyx is punctured in a controlled and predictable fashion. These are the access techniques most commonly used by urologists. However, gaining access to a pre-identified calyx is usually the most difficult part of PCNL. It often requires multiple needle punctures and prolonged radiological screening, and sub-optimal access leads to increased operative times and decreased stone-free rates.

Robotic assisted access: The first robotic system to access the renal collecting system for PCNL was described by Potamianos et al. in 1995 and consisted of a manually positioned robotic arm mounted on the operating table, guided by C-arm fluoroscopy $(5,6)$. Cadeddu et al. designed a fully automated robot that managed all aspects of PC access: planning the needle trajectory, needle positioning and advancement and real-time needle tracking using biplanar fluoroscopy (7). Stoianovici et al. developed a metal arm with 6 degrees of freedom movement that was manipulated mechanically by the urologist. It was attached to the side rail of the operating table, and incorporated a radiolucent needle grasping disc at the distal end of the arm (8). The PAKY (percutaneous access to the kidney) system and later PAKY-RCM (remote center of motion) system were improvements on the original design: the passive robotic arm was improved by adding an electronic needle insertion device and the ability to position the needle by remote control $(9,10)$.

In all the abovementioned designs, except the fully automated robot, the "robotic arms" mainly serve to stabilize and in some cases advance the needle, and reduce radiation exposure. However, the urologist still needs to calculate and plan the needle trajectory to the desired renal calyx.

Therefore, the challenge is to develop a system that is cost-effective and simple to use, yet faster and more accurate than is possible for the average general urologist. Prototypes of a fully automated robot managing all aspects of access for PCNL have been built, but their size and complexity have prevented application in routine clinical practice (7).

The aim of this study was to design a simple and cost-effective system for use by urologists to gain rapid and accurate percutaneous access for performing PCNL. Although an automated system provides rapid needle alignment and insertion, active components increase system costs. Furthermore, bi-planar fluoroscopy is not as commonly used in operating rooms as mobile $\mathrm{C}$-arm systems. This paper describes the development of a computer directed gantry system using $\mathrm{C}$-arm fluoroscopy to gain rapid and accurate renal access during PCNL.

\section{MATERIALS AND METHODS}

This access system is based on the principle of triangulation - the process of localizing a point in 3 dimensions by using 2 intersecting lines. In the setting of PCNL, the fluoroscopic images of the contrast-filled renal calyceal system obtained intra-operatively are used. One image of the collecting system is obtained with the $\mathrm{C}$-arm in the 0 -degrees position (directly perpendicular to the kidney) and the other with the C-arm tilted 20-degrees. The images are taken at the same time in the respiratory cycle during controlled mechanical ventilation.

These images are relayed to a laptop computer containing the software and graphic user interface. The ideal setup would be a system where the $\mathrm{C}$-arm has an output connected to the laptop so that the images can be transferred there directly. Due to limitations of our C-arm unit, this was unfortunately not possible and images had to be saved on a "flash drive" and manually imported to the graphic user interface on the laptop. On these images, the urologist marks the specific area of the specific calyx targeted 
for puncturing by clicking with the computer mouse on the fluoroscopic images. The software calculates the needle trajectory and subsequently provides the numerical settings for the 3 axes of the gantry, which is attached to the operating table. The needle positioning device on the gantry is set into position by the urologist according to the values provided by the software. The needle is now placed through the needle guide and inserted to the depth calculated by the software, which should result in puncturing of the targeted calyx.

\section{Hardware}

\section{Needle Positioning Device}

The central feature of this design is a needle alignment device and portable, light-weight mechanical gantry that is attached to the edge of the operating table (Figure-1). The gantry contains no motorized parts or electronic components. The system is manually adjusted by the urologist and consists of 3 orthogonal axes and a gyro-like end-effector resulting in a 6 degrees of freedom system.

The vertical axis of the system carries the combined weight of the other axes and end-effector,

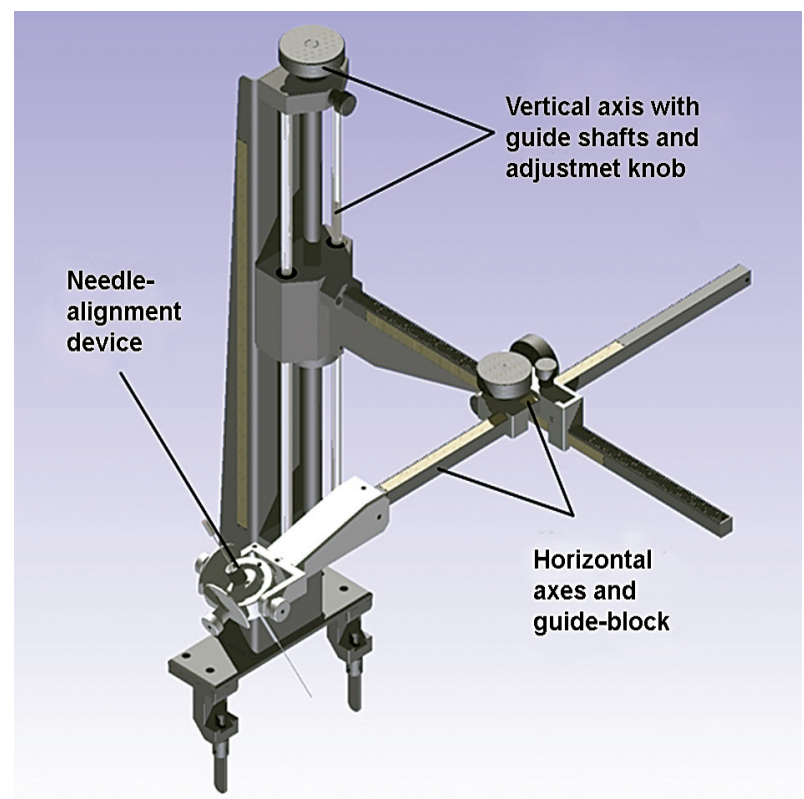

Figure 1 - Mechanical gantry with needle alignment device. and is attached to the existing rails on the theatre table. High precision guide shafts with closed linear bushings ensure accuracy. An adjustment resolution of 2 $\mathrm{mm}$ per knob rotation is achieved. A vertical translation range of $400 \mathrm{~mm}$ is possible, which is more than adequate to accommodate patients with varying body habitus.

A rack and pinion option provides flexibility to the design and simplifies placement of fixing knobs for the horizontal axes. Bending and torsion forces are addressed by a web and a counter balance, which prevents twisting of the rack. A guide-block, housing the pinions, adjustment- and fixing knobs, ensures high precision linear movement of the 2 rack and pinion configurations. The horizontal axes cover a $450 \mathrm{~mm}$ $\mathrm{x} 450 \mathrm{~mm}$ area at the height of the vertical axis.

The gyro mechanism or needle-alignment mechanism (end-effector) orientates the needle around a fixed point (Figure-2). Aligning a needle with a specified vector requires 2 rotational degrees of freedom. As the needle-alignment mechanism will be positioned over the kidney, a radiolucent acrylic (Perspex) was used in the manufacturing of the mechanism. It consists of 3 main components for the 2 axes of rotation: a fixed base, an outside ring for rotation around the $y$-axis and an inside ring for rotation around the $\mathrm{x}$-axis. The rings are locked in

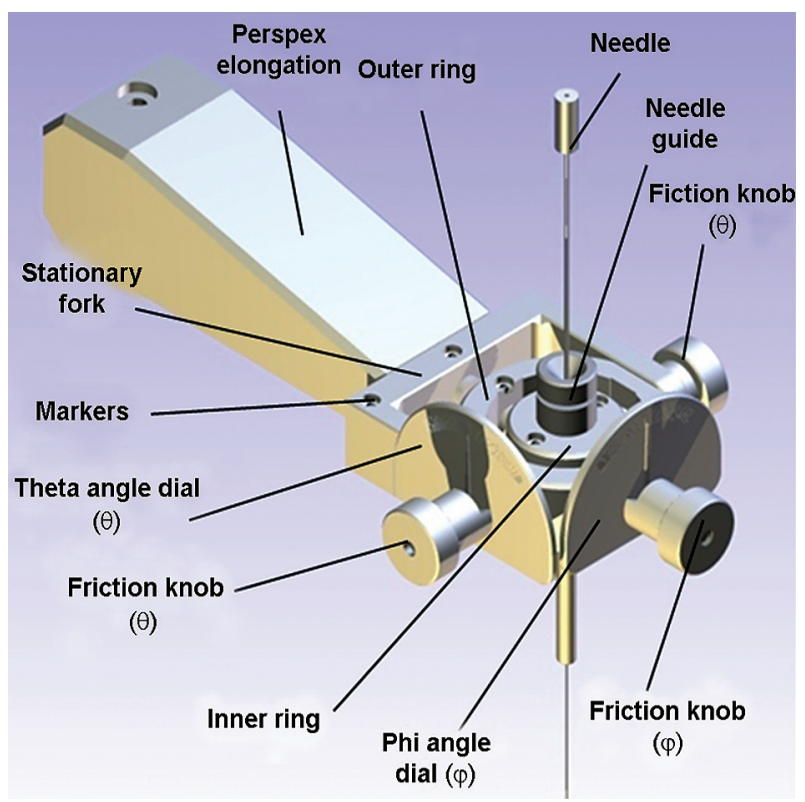

Figure 2 - Needle alignment device with needle inserted. 


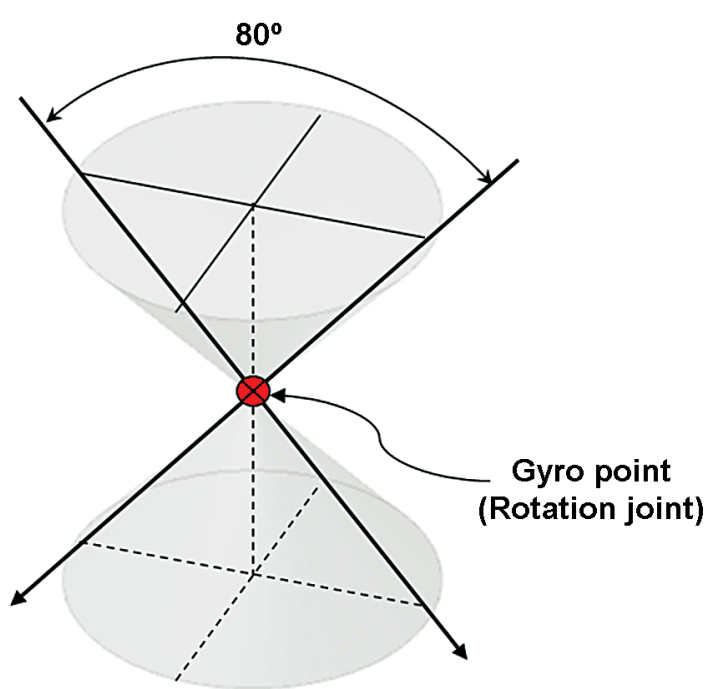

Figure 3 - Movement volume of needle in the gyro mechanism (the gyro point is the targeted point used for translational calculations).

position by friction locks. Engraved dials display the angle of needle rotation, which ranges \pm 40 -degrees around both axes, resulting in the needle rotation range shown in Figure-3.

Two groups of $2 \mathrm{~mm}$ diameter stainless steel navigation markers, required for coordinate identification and needle manipulation, are located on the radiolucent needle-alignment mechanism. These are called the gantry and needle marker groups. The needle is gripped by a friction mechanism, which allows the urologist to adjust the friction by which the needle is held during insertion.

\section{Software}

Software was designed using Python ${ }^{\circledR}$ (which can be obtained via free internet download) and was run on a standard laptop computer.

User interface - The control center of the positioning system is the urologist-operated user interface, aiding in the calibration and the targeting procedure.

Calibration screen - The user is required to select and import the calibration images. The calibration algorithm is performed automatically and takes approximately 15 seconds to complete.

Point selection screen - All points on the specified calyx required for targeting are identified and selected by the urologist - this provides the calyx markers.

Targeting screen - This shows the final translation and rotation (i.e. numerical settings of the 3 axes of the gantry) required for targeting.

- Insertion routines - Two insertion routines can be utilized with the information gained (Figure-4).

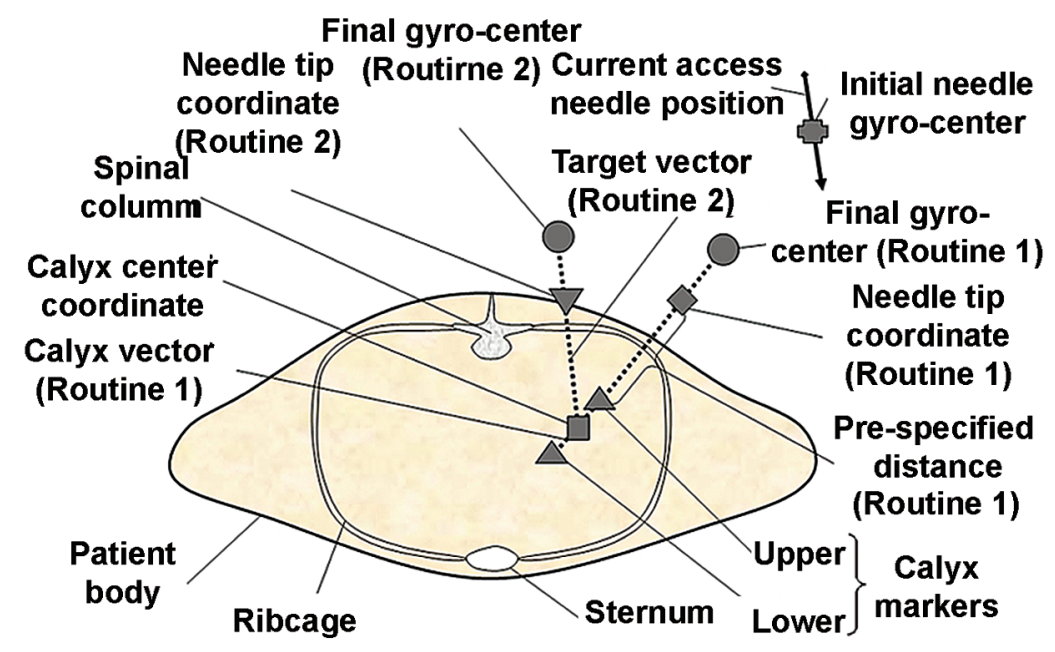

Figure 4 -Illustration of prone patient, showing the 2 insertion procedures. 


\section{Computer Assisted System for Calyceal Access in PCNL}
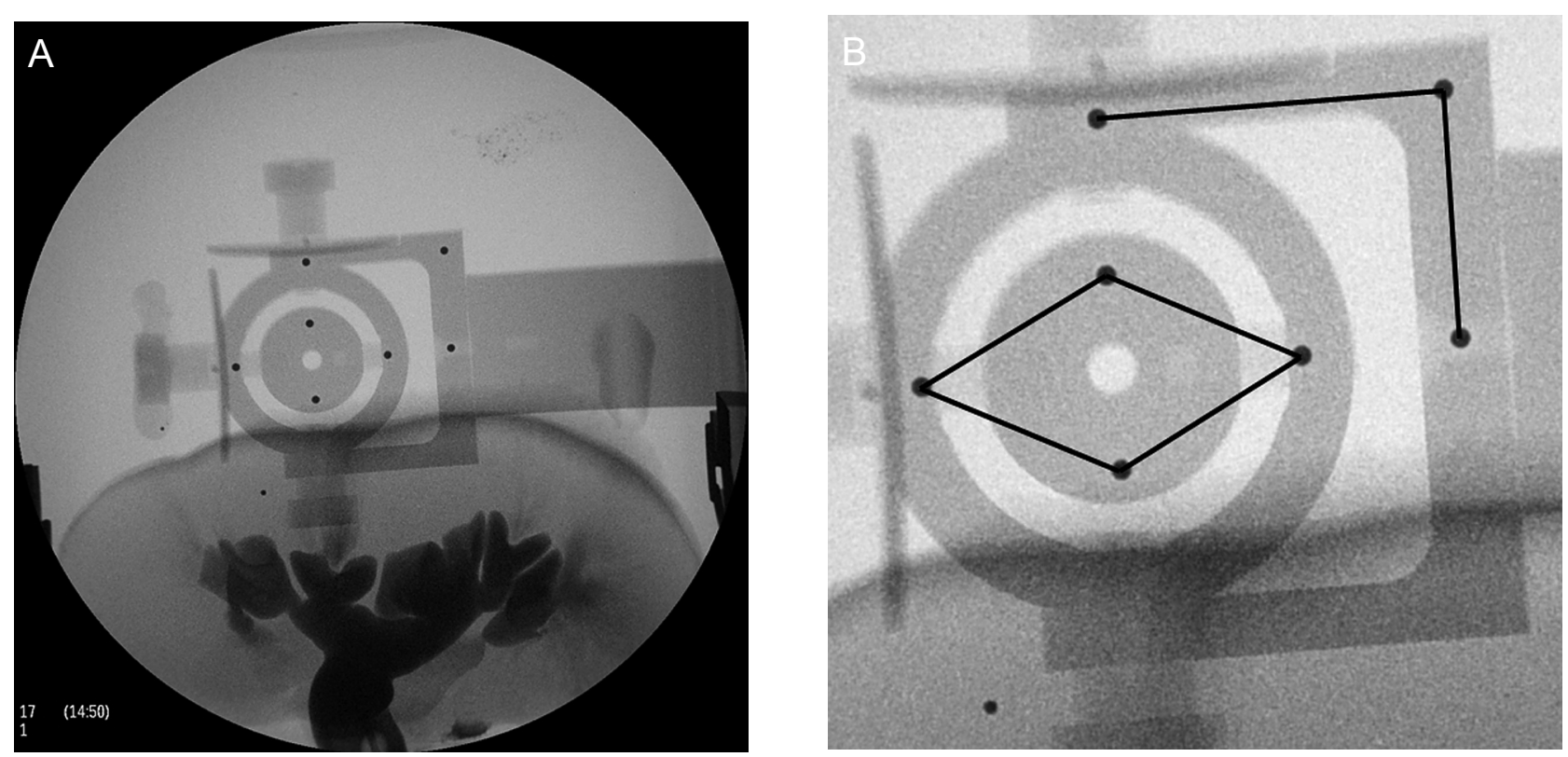

Figure - 5 A) Fluoroscopic image of needle positioner and contrast-filled porcine kidney. B) Enlargement of A, showing needle markers (diamond shape), gantry markers (L-shape) and access marker (dot at bottom left).

- Insertion routine 1 uses the defined calyx vector and needle tip coordinate and attempts to orientate the needle in this vector at a pre-specified distance from the calyx tip (Figure-4). Routine 1 resembles the "triangulation" technique.

- Insertion routine 2 uses the needle tip coordinate and calyx center coordinate to provide a new vector to which the needle is adjusted (Figure-4). Routine 2 resembles the "eye of the needle" technique.

As the targeted gyro-center coordinates for the two respective insertion routines are known, the required translation from the initial gyro-center coordinate to any of the targeted markers can be computed. The operator can select either one of the two insertion routines. The required translation in the gantry $\mathrm{x}-, \mathrm{y}-, \mathrm{z}$ directions for the respective routines is calculated as the difference between the transformed coordinates of the target and initial gyro mechanism center coordinates:

Marker Selection - Three groups of navigation markers require selection (Figure-5). They are called the gantry, needle and access marker groups, respectively. The needle markers are visible as a diamond shaped configuration. The gantry markers on their outside are in the shape of a flipped capital letter "L". The access marker shows as a small individual artifact which is easily recognized (Figure-5B). The needle markers serve a dual function: they define the needle orientation and the center of rotation of the gyro mechanism. The gantry markers define the

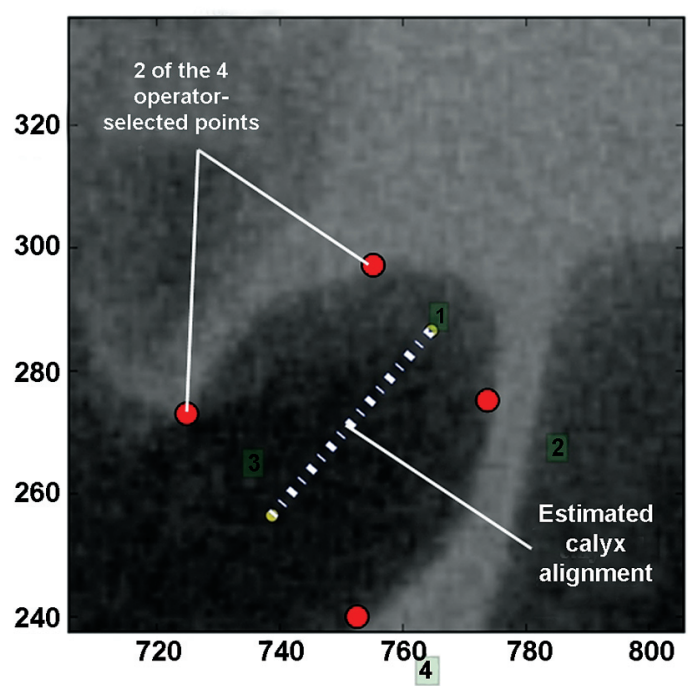

Figure 6 - Enlarged view of the 0-degrees image (as seen by the operator on the user interface) in which the 4-point method has been used - 4 points on the edges of the desired calyx have been selected. 
movement directions of the $\mathrm{x}, \mathrm{y}$ and $\mathrm{z}$ translations of the needle positioning system. The access marker defines the needle access point.

Determining point correspondences of the kidney calyx in the stereo image pair is problematic, as definite corresponding structures or points are not easily discerned. A four or two-point selection method can be implemented (Figure-6). In the four-point method, 2 points are selected at either end of one edge of the calyx, and another 2 on the opposite edge of the calyx. The calyx vector is determined by triangulation and subtraction of the coordinates halfway between the 2 selected calyx-end coordinates (Figure-6). With the two-point method, a point is selected centrally at the one end of the calyx and another on the opposite end in the estimated center of the calyx.

It was found that the four-point method reduced the point selection error in cases where calyx image edges were unclear (due to limitations of the fluoroscopy system). In cases where a distinct edge could be identified, the two-point method was adequate.

\section{Experimental Setup for Testing}

\section{Laboratory Testing}

The operating room environment was simulated using digital cameras in a configuration resembling that of the C-arm fluoroscopy system (Figure7). This allowed thorough testing of the system in a controlled environment to determine mechanical design accuracy and repeatability. This setup also

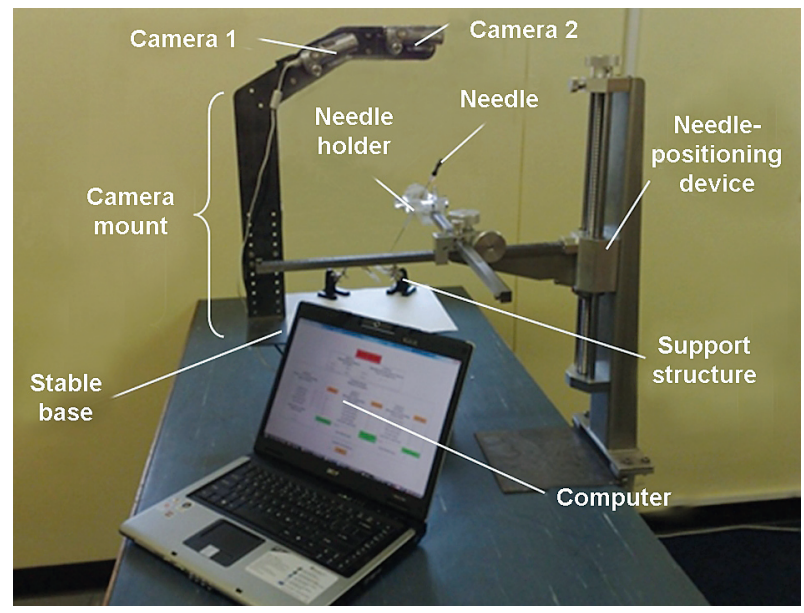

Figure 7 - Laboratory experimental testing setup.

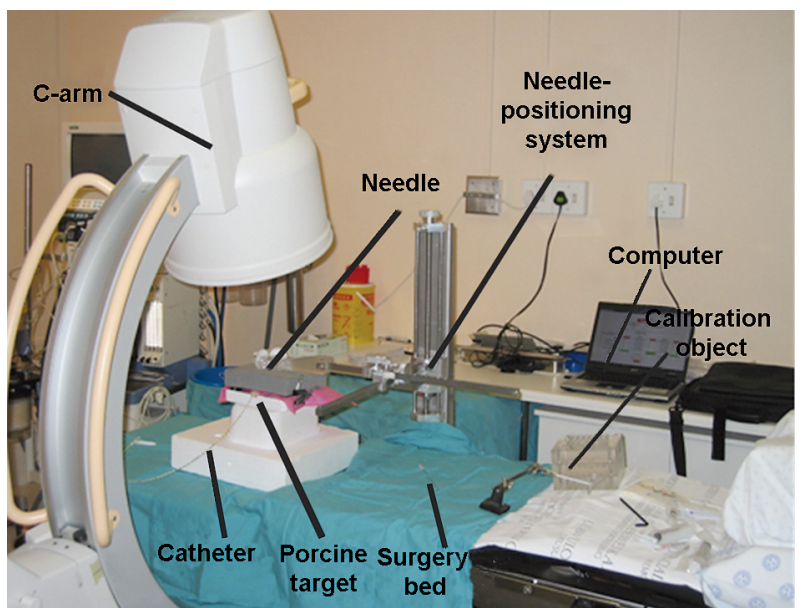

Figure 8 - Operating room experimental setup using contrastfilled porcine kidney.

allowed a basis for algorithm testing during system development stages.

\section{Operating Room Testing}

Testing of the system using C-arm fluoroscopic imaging was performed on 2 target types in an operating room (Figure-8).

Target type 1 consisted of radiolucent acrylic plastic tubes, 5 and $10 \mathrm{~mm}$ in diameter (approximate calyceal diameter) and $30 \mathrm{~mm}$ in length, with two 1 $\mathrm{mm}$ diameter stainless steel spheres attached on opposite sides of each tube.

Target type 2 consisted of a foam-occluded, contrast-filled porcine kidney - resembling a model described by De Sa Earp for teaching PCNL access (11). An occlusion balloon catheter was inserted into the ureter and blue-colored radiological contrast medium introduced into the collecting system under gravitational force.

Apart from the imaging system and target differences, the same steps used during the laboratory setup were followed. The operating room experimental setup is shown in Figure-8.

\section{RESULTS}

The cost of manufacturing the gantry and needle positioning mechanism was approximately US\$1,500. All other equipment (operating table, C- 

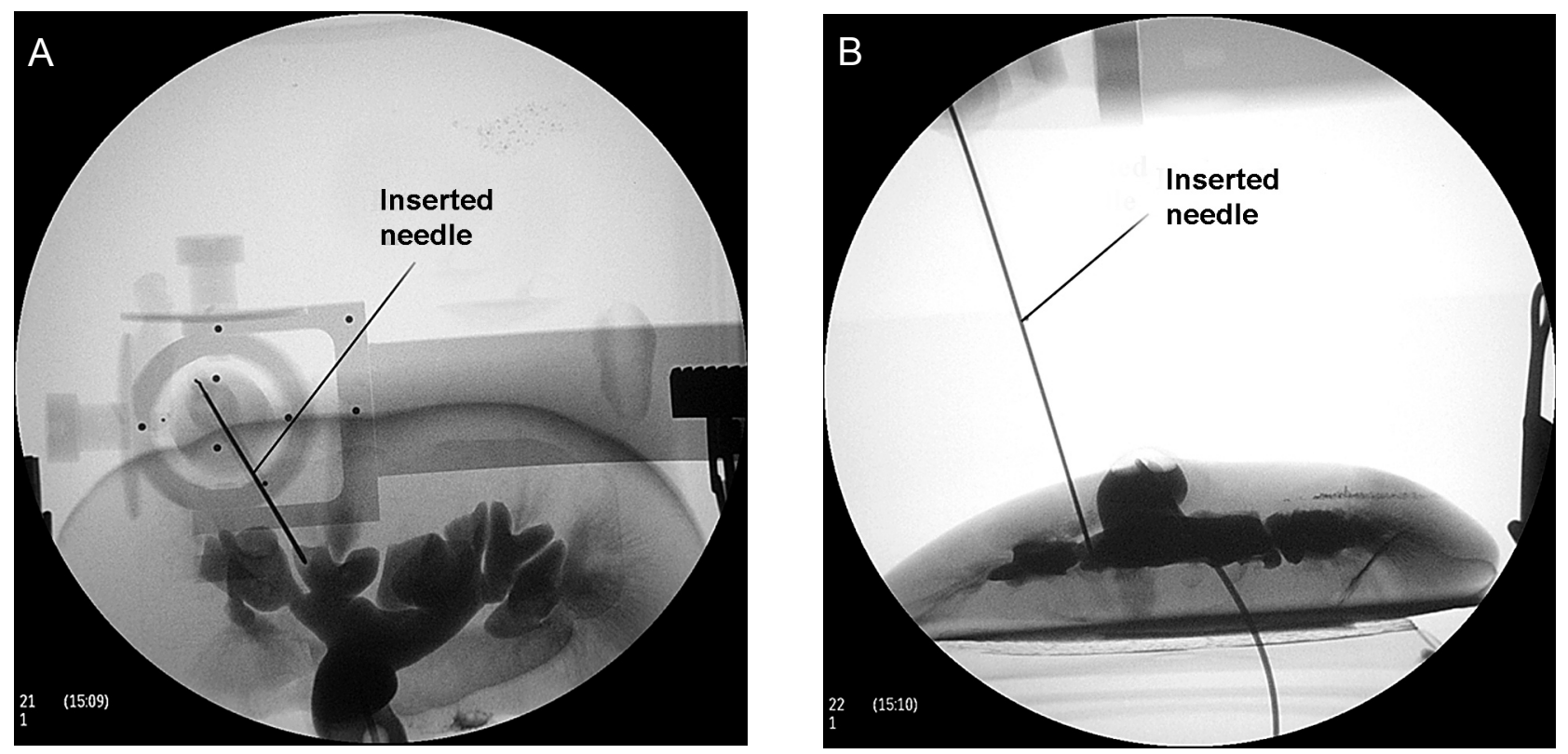

Figure $9-$ A) Fluoroscopic image at 0-degrees indicating needle alignment with the targeted calyx, and B) at 90-degrees showing needle insertion depth.

arm fluoroscopy, access needle) were standard as for routine PCNL.

Fourteen tests were performed in the operating room using the $10 \mathrm{~mm}$ diameter target type-1. Targeting done within $50 \mathrm{~mm}$ above and $50 \mathrm{~mm}$ below the calibrated volume resulted in a $100 \%$ targeting success rate. Due to the large distortion in the X-ray images, reconstruction of points too far outside the calibrated volume resulted in large targeting errors.

Seven tests completed with the $5 \mathrm{~mm}$ diameter target type-1 resulted in the same success rate. The targeting procedure took approximately 10 minutes to complete for each of the respective type- 1 targets.

Two target type- 2 procedures were performed. Successful needle insertion was achieved in both cases and validated by aspiration of blue contrast medium through the needle as well as fluoroscopic screening (Figure-9). The targeting procedure for the type-2 targets took approximately 15 minutes to complete, excluding the calibration process.

\section{COMMENTS}

PCNL is the most difficult stone surgery technique for the trainee urologist to learn (12). PC access performed by a radiologist is a practical option, often employed. However, PC access related complications are fewer and stone-free rates are higher with urologist-acquired access $(3,13)$.

Training on animals is difficult to organize, expensive and not sufficiently close to human reality. Häcker et al. described teaching ultrasound- or fluoroscopy guided PC renal access with a porcine kidney "hidden" in a chicken carcass (14). Various virtual reality and inanimate simulators for endourological training have been developed, but none specifically for PC renal access (15).

Heyns and Van Gelderen (1990) were the first to propose computed tomography imaging of the pelviocalyceal system with 3-dimensional reconstruction as an aid in selecting the appropriate calyx for PCNL (16). Mozer et al. superimposed ultrasound images onto fluoroscopic images to help plan PC renal puncture (17). "Pelviocalyceal biomodeling" (18) and "rapid prototyping" (19) have been described to create a replica of individual patients' stone-containing renal collecting systems, on which PCNL and PC access can be planned and practiced prior to the actual surgery.

The robots and robotic arms discussed earlier are certainly accurate in obtaining access. 
Unfortunately, they are still too large and/or expensive. Furthermore, the majority of these systems still rely on the urologist to calculate the trajectory of the needle to the desired calyx. Robots for PCNL have been used in experimental settings only. Obtaining remote $\mathrm{PC}$ renal access using an automated telesurgical robotic system has been successfully performed (20). With this system, an experienced endourologist is only a phone call away. This holds promise for the future.

\section{CONCLUSIONS}

The mechanical gantry system described in this paper is low-cost, portable, light-weight, sturdy and resilient. C-arm fluoroscopy is limited to two images, thus reducing radiation exposure significantly. As there are no electronic switches or dials involved in positioning the gantry and needle alignment mechanism, it is simple to set up and operate. Testing of the system, both in the laboratory and in the operating room with the use of contrast filled porcine kidneys, showed an extremely high degree of accuracy in gaining precise access to a targeted renal calyx. Further study is required to validate its use during PCNL in humans.

Further study is also required to improve some engineering and design features. As C-arm imaging systems were not designed to produce stereoscopic images for point triangulation, errors due to movement during the acquisition of targeting images are expected. A method to accurately monitor and control C-arm positioning is necessary. Another problem in image-guided systems requiring calibration prior to targeting is the fact that only a specified volume is calibrated. Point reconstruction outside this volume introduces large errors. Factors such as needle deflection due to tissue resistance and obstruction by adjacent structures were not addressed at this stage of development.

\section{ACKNOWLEDGEMENTS}

Research funding for this project was provided by the Urological Association of South Africa.
The authors would like to thank Malcolm April, Elsabé du Toit and Dr Willem Groenewald from the Department of Medical Imaging and Clinical Oncology at Tygerberg Hospital for their assistance and the use of the BV Pulsera fluoroscopy system.

The University of Stellenbosch, on behalf of the authors, is the intellectual property owner of the design, manufacture and patent rights of the equipment described in this paper.

\section{CONFLICT OF INTEREST}

None declared.

\section{REFERENCES}

1. Fernström I, Johansson B: Percutaneous pyelolithotomy. A new extraction technique. Scand J Urol Nephrol. 1976; 10: 257-9.

2. Miller NL, Matlaga BR, Lingeman JE: Techniques for fluoroscopic percutaneous renal access. J Urol. 2007; 178: 15-23.

3. Watterson JD, Soon S, Jana K: Access related complications during percutaneous nephrolithotomy: urology versus radiology at a single academic institution. $\mathrm{J}$ Urol. 2006; 176: 142-5.

4. Lawson RK, Murphy JB, Taylor AJ, Jacobs SC: Retrograde method for percutaneous access to kidney. Urology. 1983; 22: 580-2.

5. Potamianos P, Davies BL, Hibberd RD: Intra-operative imaging guidance for keyhole surgery: methodology and calibration. Proceedings of the First International Symposium on Medical Robotics and Computer Assisted Surgery, Pittsburgh, Pennsylvania. 1994; pp. 98-104.

6. Potamianos P, Davies BL, Hibberd RD: Intra-operative registration for percutaneous surgery. Proceedings of the Second International Symposium on Medical Robotics and Computer Assisted Surgery, Baltimore, Maryland. 1995; pp. 156-64.

7. Cadeddu JA, Bzostek A, Schreiner S, Barnes AC, Roberts WW, Anderson JH, et al.: A robotic system for percutaneous renal access. J Urol. 1997; 158: 158993.

8. Stoianovici D, Cadeddu JA, Demaree RD, Basile SA, Taylor RH, Whitcomb LL at al.: An efficient needle injection technique and radiological guidance method for percutaneous procedures. Proc 1st Joint Conf 
CRVMed II \& MRCAS III, Grenoble, France. 1997; pp. 295-8.

9. Cadeddu JA, Stoianovici D, Chen RN, Moore RG, Kavoussi LR: Stereotactic mechanical percutaneous renal access. J Endourol. 1998; 12: 121-5.

10. Su LM, Stoianovici D, Jarrett TW, Patriciu A, Roberts WW, Cadeddu JA, et al.: Robotic percutaneous access to the kidney: comparison with standard manual access. J Endourol. 2002; 16: 471-5.

11. Earp PP: Percutaneous renal surgery--new model for learning and training. Int Braz J Urol. 2003; 29: 1514.

12. Tanriverdi O, Boylu U, Kendirci M, Kadihasanoglu M, Horasanli K, Miroglu C: The learning curve in the training of percutaneous nephrolithotomy. Eur Urol. 2007; 52: 206-11.

13. Osman M, Wendt-Nordahl G, Heger K, Michel MS, Alken P, Knoll T: Percutaneous nephrolithotomy with ultrasonography-guided renal access: experience from over 300 cases. BJU Int. 2005; 96: 875-8.

14. Häcker A, Wendt-Nordahl G, Honeck P, Michel MS, Alken P, Knoll T: A biological model to teach percutaneous nephrolithotomy technique with ultrasound- and fluoroscopy-guided access. J Endourol. 2007; 21: 545-50.

15. Laguna MP, Hatzinger M, Rassweiler J: Simulators and endourological training. Curr Opin Urol. 2002; 12: 209-15.

16. Heyns CF, van Gelderen WF: 3-dimensional imaging of the pelviocaliceal system by computerized tomographic reconstruction. J Urol. 1990; 144: 1335-8.

17. Mozer P, Conort P, Leroy A, Baumann M, Payan Y, Troccaz J, et al.: Aid to percutaneous renal access by virtual projection of the ultrasound puncture tract onto fluoroscopic images. J Endourol. 2007; 21: 460-5.

18. Radecka E, Brehmer M, Holmgren K, Palm G, Magnusson P, Magnusson A: Pelvicaliceal biomodeling as an aid to achieving optimal access in percutaneous nephrolithotripsy. J Endourol. 2006; 20: 92-101.

19. Bruyère $F$, Leroux $C$, Brunereau $L$, Lermusiaux $P$ : Rapid prototyping model for percutaneous nephrolithotomy training. J Endourol. 2008; 22: 91-6.

20. Bauer J, Lee BR, Stoianovici D, Bishoff JT, Micali S, Micali F, et al.: Remote percutaneous renal access using a new automated telesurgical robotic system. Telemed J E Health. 2001; 7: 341-6.

Accepted after revision:

May 7, 2010

\section{Correspondence address:}

Dr. A. D. Zarrabi

Department of Urology

University of Stellenbosch and Tygerberg Hospital

PO Box 19063, Tygerberg, 7505, South Africa

Fax: + 2721 933-8010

E-mail: adzarrabi@gmail.com

\section{EDITORIAL COMMENT}

This article proposes an interesting method for gaining the calyx in a percutaneous surgery and offers some important aspects like a welcoming less exposure to irradiation, and requires less expertise of the surgeon for accessing the urinary tract.
Since of beginning of percutaneous nephrolithotripsy it is well known that the puncture of the urinary system is best done by the urologist. He chooses the calyx that could offer the best access to the determined surgery. When an other access be- 
comes necessary in a more complex surgery, the surgeon without any other assistance can perform the procedure.

However, in several services the urinary tract approach continues to be done by invasive radiologists. For novice urologists and for those who are ra- diologist dependent, the equipment and the software may be useful since it could be really cost-effective, commercially available and clinically tested. It is an additional way to give autonomy to urologists in percutaneous surgery.

Dr. Anuar I. Mitre

Division of Urology

University of Sao Paulo, USP

Sao Paulo, SP, Brazil

E-mail:anuar@mitre.com.br

\section{EDITORIAL COMMENT}

Undoubtedly, percutaneous surgery for kidney stones is the most demanding procedure for a young endourologist to learn. As a matter of fact, it is also cumbersome for the expert endourologist to pass onto the apprentice the techniques of the procedure in a stepwise and clear manner. Having said that, any effort to unfold the tricks of this treatment modality is worth trying.

Simulators, animal models, "ex vivo" and "in vivo" training models are being built and tested worldwide, but to date there has not been a single one that has proven capable of reproducing the "real life" challenges of the percutaneous nephrolithotomy. The present study creates a fixed computerized geometrical concept of the procedure, where land- marks are delimitated and loaded onto a computer that generates angles and aligns the tip of the needle towards the target (chosen calyx). The idea is very interesting and minimizes the mistakes of the human made puncture, but it needs further testing in "in vivo" animal models.

However, understanding the mechanism on which this study was based is the first and most important step for performing a safe calyx puncture. Basically, the triangulation technique has been reproduced by the authors, and once one (reader) understands this concept, the puncture becomes less enigmatical.

\section{Dr. Renato Nardi Pedro \& Dr. Nelson Rodrigues Netto Junior Division of Urology, UNICAMP \\ Sao Paulo, SP, Brazil E-mail: rnpedro@unicamp.br}

\section{EDITORIAL COMMENT}

Nephrolithiasis is a worldwide problem that accounts for significant morbidity and expense. Indications for an active treatment of renal calculi go mainly according to the stone size as well as the clinical symptoms. The goal of Nephrolithiasis treatment is the complete removal of all stones from the pelviocalyceal system with the lowest possible morbidity. Extracorporeal shockwave lithotripsy, percu- 
taneous nephrolitholapaxy and flexible ureterorenoscopy are the main options for treatment.

Percutaneous nephrolithotomy has undergone an evolution in technique and in equipment since its introduction in the late 1970s. This evolution continues today and is evidenced by the numerous publications about the technique.

Although ureteroscopy and shock wave lithotripsy predominate in the treatment of urolithiasis, percutaneous nephrolithotomy continues to be an important part of the urologist's armamentarium. Percutaneous nephrolithotomy is a minimally invasive surgery that causes minimal renal injury and maximizes stone clearance, especially in patients with complex stone disease.
The authors in this paper design a simple, costeffective system for gaining rapid and accurate calyceal access during percutaneous nephrolithotomy.

They showed that with the system described, we can have a new project with low-cost $(\$ 1500)$, portable, light-weight, that can help any other urologist around the world.

It is very important that the $\mathrm{C}$-arm fluoroscopy is limited to two images, thus reducing the risk of all Urologists who live with radiation exposure.

As the authors concluded, "Further study is required to validate its use during PCNL in the human" and improve the system. We will be expecting the human results.

Dr. Mauricio Rubinstein Section of Urology Federal University of Rio de Janeiro State Rio de Janeiro, RJ, Brazil E-mail:mrubins74@hotmail.com

\section{EDITORIAL COMMENT}

The authors are to be congratulated on their innovative work aimed at developing a reliable and simple technology to aid with accurate calyceal access during PCNL. Many urologists in the United States rely on radiologist to gain initial kidney access at the time of surgery. I hope that continued development of this technology or something similar that is inexpensive may help urologists gain their own precise access. Any excitement for this innovation must be tempered, however, by the lack of in vivo testing where muscle and fascial deformation as well as kidney movement may diminish calyceal puncture success. We await further animal and clinical reliability evaluations.

Dr. Jeffrey Anthony Cadeddu Department of Urology UT Southwestern Medical Center Dallas, Texas, USA E-mail: jeffrey.cadeddu@utsouthwestern.edu 